Reprod. Nutr. Dévelop., 1987, 27 (1 B), 325-326.

\title{
Données préliminaires sur l'évolution nycthémérale des dépenses énergétiques du jeune poulain
}

\author{
M. VERMOREL, J. VERNET, W. MARTIN-ROSSET (*) \\ Laboratoire d'Etude du Métabolisme Energétique, \\ (*) Unité Elevage et Alimentation du Cheval, \\ I.N.R.A. Theix, 63122 Ceyrat, France.
}

\begin{abstract}
Summary. Heat production of a young foal was continuously measured by indirect calorimetry ; energy balances were determined over 2 periods of 3 consecutive days : at 83 (P1) and 132 (P2) days of age. In P1, the foal was given only milk substitute and heat production increased by $5 \%$ after each of the 3 meals, whilst its resporatory quotient (QR) increased from 0.82 to 0.96 after the morning meal. In P2, when milk substitute, hay and concentrate were fed, heat production iricreased by 12 or $20 \%$ after the two meals (fig. 1). The results have been compared with those previously obtained in calves fed similar diets at the same feeding levels.
\end{abstract}

II n'existe pas, à notre connaissance, de données sur le métabolisme énergétique du poulain. Nous avons donc profité de la présence d'un poulain orphelin élevé en allaitement artificiel pour obtenir une première information en vue d'une étude ultérieure sur l'utilisation de l'énergie par le poulain en croissance.

Matériel et méthodes. Les mesures ont été effectuées sur un seul animal aux âges de 83 jours (période $\mathrm{P} 1$ ) et de 132 jours (P2) et aux poids de 203 et $284 \mathrm{~kg}$ respectivement. En $\mathrm{P} 1$, le poulain recevait uniquement $24 \mathrm{~kg}$ de lait de remplacement $13120 \mathrm{~g}$ de poudre à $19 \%$ de matières grasses et $22 \%$ de matières azotées) en 3 repas égaux distribués à $9 \mathrm{~h}, 14 \mathrm{~h}$ et $20 \mathrm{~h}$. En P2, la ration était composée de 12 I du même lait ( $1800 \mathrm{~g}$ de poudre), $0,9 \mathrm{~kg}$ de foin et $4 \mathrm{~kg}$ d'aliment concentré, et distribuée en 2 repas égaux à $9 \mathrm{~h}$ et $16 \mathrm{~h} 30$. La production de chaleur du poulain a été déterminée en continu pendant 3 jours consécutifs par calorimétrie indirecte. Les résultats sont comparés à ceux obtenus précédemment avec des veaux âgés de 3 ou 5 mois, pesant 137 ou $240 \mathrm{~kg}$, réalisant des gains de poids de 1,16 ou 1,3 kg par jour et recevant des rations similaires (Vermorel et al., 1974, 1980).

Résultats et discussion. Le poulain a eu un gain de poids élevé : 1,9 et $1,8 \mathrm{~kg} / \mathrm{j}$ au cours de $\mathrm{P} 1$ et $\mathrm{P} 2$ respectivement. II a ingéré en moyenne 15,37 et $27,19 \mathrm{Mcal} / \mathrm{j}$ d'énergie brute, 13,13 et 19,89 Mcal d'énergie métabolisable (EM), a produit 8,29 et $13,73 \mathrm{Mcal}$ de chaleur et fixé 4,84 et $6,16 \mathrm{Mcal} / \mathrm{j}$ soit 2,6 et 3,4 Mcal par $\mathrm{kg}$ de gain de poids vif pendant ces 2 périodes.

En P1, la production de chaleur du poulain, associée à une fixation de protéines élevée, a été supérieure de $15 \%$ à celle des veaux préruminants $(154$ contre $\left.134 \mathrm{kcal} / \mathrm{kg} \mathrm{P}^{0,75},{ }^{*}\right)$ pour des ingestions voisines d'EM (244 contre $249 \mathrm{kcal}^{*}$ ). Elle a peu varié au cours de la journée, augmentant de $5 \pm 2 \%$ après chacun dés 3 repas, contre 12 et $17 \%$ après les 2 repas chez les veaux préruminants (fig. 1). En revanche, le quotient respiratoire $(\overline{\mathrm{Q} R})$ a beaucoup plus varié que celui des veaux : de 0,82 (contre 0,93 ) à jeun, il est passé à 0,96 (contre 1,01) au cours des $5 \mathrm{~h}$ suivant le premier repas. II s'est maintenu entre 0,96 et 0,92 jusqu'à $22 \mathrm{~h}$, en raison probablement de l'ingestion rapprochée des 2 autres repas, puis il a diminué plus fortement que chez les veaux. Ces différences pourraient provenir de la 
dépense énergétique plus élevée du poulain et d'une vidange gastrique plus rapide entraînant une mobilisation intense des réserves lipidiques corporelles au cours de la nuit.
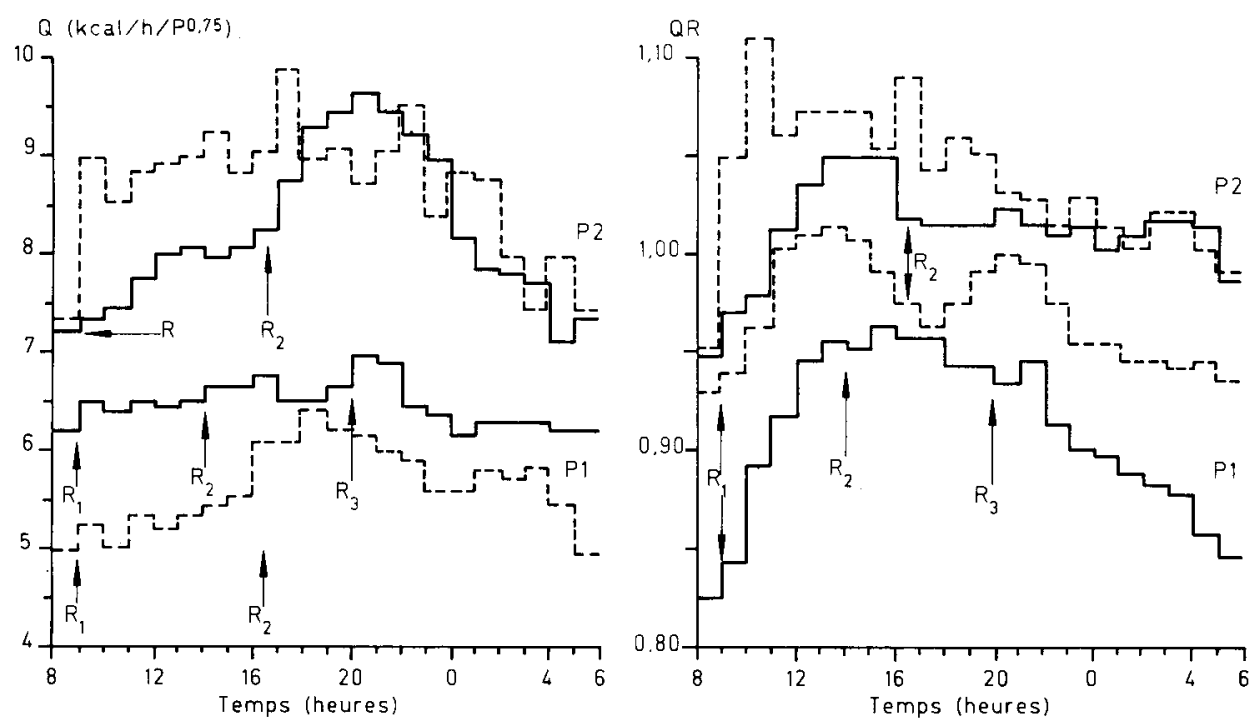

FIG. 1. - Variations, de la production de chaleur (Q) et du quotient respiratoire (QR) du poulain au cours des périodes $P 1$ et $P 2$. Comparaison aux valeurs obtenues avec des veaux (-.-) recevant des régimes similaires aux mêmes niveaux $d^{\prime}$ alimentation $(R=$ repas).

En P2, la production de chaleur du poulain a été inférieure de $6 \%$ à celle des veaux ruminants (198 contre $210 \mathrm{kcal}^{*}$ ) pour des ingestions voisines d'EM (288 contre $292 \mathrm{kcal}^{*}$ ). Les augmentations de $Q$ et du $Q R$ du poulain ont été très progressives après le premier repas, tandis que les veaux ruminants présentaient des pics importants après les 2 repas (fig. 1). Ces différences pourraient résulter d'une digestion progressive des aliments solides chez le poulain, avec production régulière de glucose dans l'intestin grêle et d'acides gras volatils (AGV) dans le gros intestin, et d'une fermentation rapide de l'aliment concentré dans le rumen des veaux, accompagnée d'une production massive de gaz carbonique, de chaleur de fermentation et d'AGV dont l'extra-chaleur est supérieure à celle du glucose. Ces premiers résultats montrent l'intérêt d'études associées sur la digestion et le métabolisme énergétique chez le poulain avant et après sevrage.

Vermorel M., Bouvier J. C., Thivend P., Toullec R., 1974. Proc 6th Symp. Energy Metabolism, 143-146. E.A.A.P. Publ. $n^{\circ} 14$

Vermorel M., Bouvier J. C., Geay Y., 1980. Ann. Zootech., 29, 65-86. 